\title{
ESTRESSE, COPING E PRESENTEÍSMO EM ENFERMEIROS HOSPITALARES: NOTA PRÉVIA*
}

Juliane Umann ${ }^{1}$, Laura de Azevedo Guido ${ }^{2}$

RESUMO: Trata-se de um projeto de pesquisa com abordagem quantitativa, com o objetivo de verificar associações entre estresse, coping e presenteísmo em enfermeiros hospitalares. A pesquisa será realizada no Hospital Universitário de Santa Maria, com enfermeiros atuantes nas unidades da referida instituição, seguindo-se um protocolo de pesquisa que consta de caracterização individual, com variáveis sociodemográficas e funcionais, além de questionário composto por três instrumentos, para a avaliação do presenteísmo, estresse e coping. As variáveis e os itens que compõem os instrumentos serão analisados estatisticamente com o software Statistical Package for the Social Sciences (SPSS).

PALAVRAS-CHAVE: Enfermagem; Saúde do trabalhador; Estresse psicológico.

\section{STRESS, COPING AND PRESENTEEISM IN HOSPITAL NURSES: FOREWORD}

\begin{abstract}
This is a research project with a quantitative approach, aiming to examine associations between stress, coping and presenteeism in hospital nurses. The research will be conducted at the University Hospital of Santa Maria with nurses working in units of that institution. It will follow a research protocol that consists of individual characteristics, with socio-demographic and functiona, besides a questionnaire of three instruments for the assessment of presenteeism, stress and coping. The variables and the items that comprise the instruments will be analyzed statistically with the Statistical Package for the Social Sciences (SPSS).
\end{abstract}

KEYWORDS: Nursing, Occupational Health, Psychological stress.

\section{ESTRÉS, COPING Y PRESENTEÍSMO EN ENFERMEROS HOSPITALARES: NOTA PREVIA}

RESUMEN: Trata-se de un proyecto de investigación con abordaje cuantitativo, con objetivo de verificar asociaciones entre estrés, coping y presenteísmo en enfermeros hospitalares. La investigación será realizada en Hospital Universitário de Santa Maria con enfermeros actuantes en las unidades de la referida institución. Será obedecido un protocolo de investigación que con caracterización individual, con variables sociodemográficas y funcionales, además de cuestionário compuesto por tres instrumentos, para la evaluación del presenteísmo, estrés y coping. Las variables y los componentes de los instrumentos serán analizados estadísticamente con el software Statistical Package for the Social Sciences (SPSS). PALABRAS CLAVE: Enfermería; Salud del trabajador; Estrés psicológico.

*Projeto de Dissertação vinculado ao Programa de Mestrado Acadêmico em Enfermagem da Universidade Federal de Santa MariaPPGEnf-UFSM. Pesquisa financiada pela Coordenação de Aperfeiçoamento de Pessoal de Nível Superior (bolsa CAPES) e Fundo de Incentivo à Pesquisa (FIPE)

${ }^{1}$ Enfermeira. Mestranda em Enfermagem pela UFSM. Membro do Grupo de Estudos e Pesquisas Trabalho, Saúde, Educação e Enfermagem da UFSM.

${ }^{2}$ Enfermeira. Doutor em Enfermagem. Professor Adjunto do Departamento de Enfermagem da UFSM. Membro do Grupo de Estudos e Pesquisas Trabalho, Saúde, Educação e Enfermagem. Orientador.

\section{Autor correspondente:}

Juliane Umann

Universidade Federal de Santa Maria

R. Silva Jardim, 2149 - 97010-490- Santa Maria-RS-Brasil

Recebido: 07/07/10

Email: juumann@hotmail.com

Aprovado: 05/11/10 


\section{INTRODUÇÃO}

As grandes mudanças no cenário político, econômico e social ocorridas na segunda metade do século XX determinaram uma crise de paradigmas, de maneira que o processo saúde-trabalho passou a ser analisado não como um simples indicador do impacto do trabalho sobre os trabalhadores, mas como consequência da relação de produção, determinada socialmente pela dialética entre capital e trabalho( ${ }^{(1)}$. Neste contexto, a Enfermagem está presente em setores hospitalares considerados desgastantes, tanto pela carga de trabalho quanto pelas especificidades das tarefas e diversidade das funções desempenhadas.

Dessa forma, o estresse está presente no cotidiano do trabalho do enfermeiro, decorrente de fatores relacionados ao tipo de ambiente, relações interpessoais, autonomia profissional, grau de exigência e responsabilidade, e pode levar a alterações fisiológicas, emocionais e comportamentais que favorecem a diminuição da saúde e do bem estar desses profissionais $^{(2-3)}$.

Assim, a identificação do estresse ocupacional, bem como das estratégias de enfrentamento utilizadas nesse processo e a repercussão nas atividades laborais, corresponde a um dos grandes agentes de mudança, uma vez que desenvolvidas as possíveis soluções para minimizar seus efeitos, estas podem tornar o cotidiano da equipe de enfermagem mais produtivo, menos desgastante e, possivelmente, valorizá-lo mais no que se refere aos aspectos humanos e profissionais. Portanto, o objetivo desta pesquisa é verificar associações entre estresse, coping e presenteísmo em enfermeiros hospitalares.

\section{METODOLOGIA}

Estudo analítico, transversal e contemporâneo, com abordagem quantitativa, que será desenvolvido no Hospital Universitário de Santa Maria. A população do estudo será composta por 155 enfermeiros atuantes na assistência direta a pacientes críticos e potencialmente críticos da referida instituição. $\mathrm{O}$ protocolo de pesquisa consta de caracterização sociodemográfica e funcional, e o questionário composto por três instrumentos para a avaliação do presenteísmo (Questionário de Limitações no Trabalho), estresse (Inventário de estresse em enfermeiros) e coping (Escala de Coping Ocupacional). A aplicação destes instrumentos será realizada em um único momento, de forma individual, respeitando-se os preceitos éticos dispostos na Resolução n. 196/96. Para o armazenamento e organização das informações será construído um banco de dados em uma planilha eletrônica, no programa Microsoft Excel (Office 2007). As variáveis sociodemográficas e funcionais e os itens que compõem os instrumentos serão analisados estatisticamente com o software Statistical Package for the Social Sciences (SPSS - versão 17.0). A consistência interna das escalas será avaliada pelo Coeficiente Alpha de Cronbach, a fim de verificar a fidedignidade da medida a que os instrumentos se propõem.

\section{REFERÊNCIAS}

1. Dias EC, Rigotto RM, Augusto LGS, Cancio J, Hoefel MGL. Saúde ambiental e saúde do trabalhador na atenção primária à saúde, no SUS: oportunidades e desafios. Ciênc Saúde Colet. 2009;14(6):2061-70.

2. Safiano C, Sarquis LMM, Felli V, Giacomozzi L. O processo saúde-doença vivenciado pelos trabalhadores de enfermagem em uma instituição hospitalar. Cogitare Enferm. 2005;8(2):87-91.

3. Schmidt DRC, Dantas RAS, Marziale MHP, Laus AM. Estresse ocupacional entre profissionais de enfermagem do bloco cirúrgico. Texto \& Contexto Enferm. 2009;18(2):330-7. 